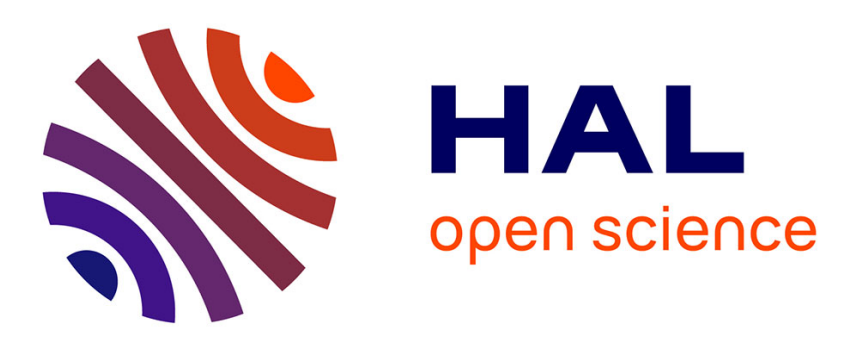

\title{
Fusion of sources of evidence with different importances and reliabilities
}

\author{
F. Smarandache, J. Dezert, J.M. Tacnet
}

\section{To cite this version:}

F. Smarandache, J. Dezert, J.M. Tacnet. Fusion of sources of evidence with different importances and reliabilities. BELIEF 2010: Workshop on the Theory of Belief Functions, Jul 2010, Edinburgh, United Kingdom. 8 p. hal-00559233

\section{HAL Id: hal-00559233 \\ https://hal.science/hal-00559233}

Submitted on 25 Jan 2011

HAL is a multi-disciplinary open access archive for the deposit and dissemination of scientific research documents, whether they are published or not. The documents may come from teaching and research institutions in France or abroad, or from public or private research centers.
L'archive ouverte pluridisciplinaire HAL, est destinée au dépôt et à la diffusion de documents scientifiques de niveau recherche, publiés ou non, émanant des établissements d'enseignement et de recherche français ou étrangers, des laboratoires publics ou privés. 


\section{Fusion of sources of evidence with different importances and reliabilities}

\author{
F. Smarandache \\ Math. \& Sciences Dept. \\ Univ. of New Mexico \\ 200 College Road. \\ Gallup, NM 87301, USA.
}

\author{
J. Dezert \\ French Aerospace Lab. \\ ONERA/DTIM/SIF \\ 29 Av. de la Div. Leclerc \\ 92320 Châtillon, France. \\ jean.dezert@onera.fr
}

\author{
J.-M. Tacnet \\ CEMAGREF \\ Unité Etna \\ 2 rue de la Papèterie, \\ 38402 St. Martin d'Hères, France. \\ jean-marc.tacnet@cemagref.fr
}

\begin{abstract}
This paper presents a new approach for combining sources of evidences with different importances and reliabilities. Usually, the combination of sources of evidences with different reliabilities is done by the classical Shafer's discounting approach. Therefore, to consider unequal importances of sources, if any, a similar reliability discounting process is generally used, making no difference between the notion of importance and reliability. In fact, in multicriteria decision context, these notions should be clearly distinguished. This paper shows how this can be done and we provide simple examples to show the differences between both solutions for managing importances and reliabilities of sources. We also discuss the possibility for mixing them in a global fusion process.
\end{abstract}

Keywords: Information fusion, DSmT, discounting, importance, reliability, AHP.

\section{Introduction}

In many real-life fusion problems, one has to deal with different sources of information arising from human reports, artificial intelligence experts systems and/or physical sensors. The information are usually imprecise, uncertain, incomplete, qualitative or quantitative and possibly conflicting. The task of information fusion is to combine all the information in such a way that one has a better understanding and assessment of the situation of the complex problem under consideration for decision-making support. Several theoretical frameworks have been proposed in the literature (Probability theory, Possibilities theory, Imprecise PT, etc) but the most appealing ones are the theories of belief functions, originally known as Dempster-Shafer Theory (DST) [8] and then extended and refined in Dezert-Smarandache Theory (DSmT) [9] for dealing with qualitative information, for fusioning highly conflicting sources of evidences, for conditioning evidences, etc. Aside the choice of the "best" rule of combination of sources of evidences characterized by their be- lief functions, more specifically by their basic belief assignments (bba's), or belief masses, the very important problem concerns the possibility that sources involved in the fusion process may not have the same reliability, neither the same importance. The reliability can be seen as an objective property of a source of evidence, whereas the importance of a source is a subjective property of a source expressed by the fusion system designer.

The reliability of a source represents its ability to provide the correct assessment/solution of the given problem. The importance of a source represents somehow the weight of importance granted to the source by the fusion system designer. The reliability and importance represent two distinct notions and the fusion process must be able to deal with these notions. We show in this paper how this can be done efficiently through two discounting techniques using Proportional Conflict Redistribution rules no 5 or no 6 (PCR5 or PCR6) developed in DSmT framework. We will show also that such solution cannot be used in DST framework using Dempster's rule of combination because Dempster's rule doesn't respond to our new importance discounting (it only responds to reliability discounting ${ }^{1}$ ).

The importance of a source is particularly crucial since it is involved in multi-criteria decision making (MCDM) problems, like in the Analytic Hierarchy Process (AHP) developed by Saaty [6, 7]. That's why it is fundamental to show how the importance can be efficiently managed in evidential reasoning approaches, in particular in DSmT. The fusion system designer is still free to make no differences between importance and reliability and use the classical discounting technique. In general however, one should consider the importance and the reliability as two distinct notions and thus they have to be processed in different ways. This is the purpose of this paper. The application of this technique in DSmT-AHP is presented in [2] and an application of both DSmT and AHP for risk expertise and prevention in mountains has been introduced by Tacnet in $[11,12]$

\footnotetext{
${ }^{1}$ Known as the classical Shafer's discounting, see [8].
} 
and works are still under progress in this field.

This paper is organized as follows. After a brief reminder of basics of DSmT for information fusion and its main fusion rule in section 2, we present the classical discounting technique for combining sources with different reliabilities in section 3 . In section 4 , we introduce in a new solution for taking into account the possible different importances of sources in the fusion process. Section 5 provides simple examples to show and compare the results obtained from the two discounting approaches. In section 6 we discuss the more general problem where one needs to deal with both reliability and importance at the same level in the fusion process. Conclusions and perspectives are given in section 7 .

\section{Basics of DSmT}

Let $\Theta=\left\{\theta_{1}, \theta_{2}, \cdots, \theta_{n}\right\}$ be a finite set of $n$ elements $\theta_{i}, i=1, \ldots, n$ assumed to be exhaustive. $\Theta$ corresponds to the frame of discernment of the problem under consideration. In general (unless introducing some integrity constraints), we assume that elements of $\Theta$ are non exclusive in order to deal with vague/fuzzy and relative concepts [9], Vol. 2. This is the so-called freeDSm model. In DSmT framework, there is no need in general to work on a refined frame consisting in a discrete finite set of exclusive and exhaustive hypotheses ${ }^{2}$ because DSm rules of combination work for any models of the frame, i.e. the free DSm model (no exclusive constraint between $\theta_{i}$, Shafer's model (all $\theta_{i}$ are exclusive) or any hybrid model (only some $\theta_{i}$ are truly exclusive). The power set $2^{\Theta}$ is defined as the set of all propositions built from elements of $\Theta$ with $\cup$ [8]; $\Theta$ generates $2^{\Theta}$ under $\cup$. The hyper-power set (Dedekind's lattice) $D^{\Theta}$ is defined as the set of all propositions built from elements of $\Theta$ with $\cup$ and $\cap$; $\Theta$ generates $D^{\Theta}$ under $\cup$ and $\cap$, see [9] Vol. 1 for many detailed examples. The super-power set (Boolean algebra) $S^{\Theta}$ is defined as the set of all propositions built from elements of $\Theta$ with $U$ and $\cap$ and complement $c($.$) ; \Theta$ generates $S^{\Theta}$ under $\cup, \cap$ and $c($.$) , see [9] Vol. 3. S^{\Theta}$ can be seen as the minimal refined frame of $\Theta$. For notation convenience, we use the generic notation $G^{\Theta}$ to represent the fusion space under consideration depending on the application and the underlying model chosen for the frame $\Theta$; which can be either $G^{\Theta}$ can be either $2^{\Theta}, D^{\Theta}$ or $S^{\Theta}$. In DST framework, $G^{\Theta}=2^{\Theta}$, whereas in DSmT we usually work with $G^{\Theta}=D^{\Theta}$.

A (quantitative) basic belief assignment (bba) expressing the belief committed to the elements of $G^{\Theta}$ by a given source/body of evidence is a mapping function $m(\cdot): G^{\Theta} \rightarrow[0,1]$ such that: $m(\emptyset)=0$ and $\sum_{A \in G^{\Theta}} m(A)=1$. Elements $A \in G^{\Theta}$ having $m(A)>0$ are called focal elements of the bba $m($.$) . The general$ belief and plausibility functions are defined respectively

\footnotetext{
${ }^{2}$ Referred as Shafer's model in the literature.
}

in almost the same manner as Shafer in [8], i.e.

$$
\begin{aligned}
& \operatorname{Bel}(A)=\sum_{B \in G^{\Theta}, B \subseteq A} m(B) \\
& \operatorname{Pl}(A)=\sum_{B \in G^{\Theta}, B \cap A \neq \emptyset} m(B)
\end{aligned}
$$

In DSmT, the Proportional Conflict Redistribution Rule no. 5 (PCR5) has been proposed as a serious alternative of Dempster's rule [8] for dealing with conflicting belief functions. It has been also clearly shown in [9], Vol. 3, chap. 1 that Smets' rule ${ }^{3}$ is not so efficient, nor cogent because it doesn't respond to new information in a global or in a sequential fusion process. Indeed, very quickly Smets' fusion result commits the full mass of belief to the empty set!!! Therefore in applications, some ad-hoc numerical techniques must be used to circumvent this serious drawback. Such problem doesn't occur with PCR5 rule. By construction, other well-known rules like Dubois \& Prade, or Yager's rule, and contrariwise to PCR5, increase the non-specificity of the result. An introduction to DSmT and PCR5 fusion rule with justification and several examples can be found in [9], Vol. 3, Chap. 1, freely downloadable from the web.

Definition of PCR5 (for two sources): Let's $m_{1}($. and $m_{2}($.$) be two independent { }^{4}$ bba's, then the PCR5 rule of combination for two sources of evidence is defined as follows (see [9], Vol. 2 for details, justification and examples): $m_{P C R 5}(\emptyset)=0$ and $\forall A \in G^{\Theta} \backslash\{\emptyset\}$

$$
\begin{gathered}
m_{P C R 5}(A)=\sum_{\substack{X_{1}, X_{2} \in G^{\Theta} \\
X_{1} \cap X_{2}=A}} m_{1}\left(X_{1}\right) m_{2}\left(X_{2}\right)+ \\
\sum_{\substack{X \in G^{\Theta} \\
X \cap A=\emptyset}}\left[\frac{m_{1}(A)^{2} m_{2}(X)}{m_{1}(A)+m_{2}(X)}+\frac{m_{2}(A)^{2} m_{1}(X)}{m_{2}(A)+m_{1}(X)}\right]
\end{gathered}
$$

All fractions in (3) having zero denominators are discarded. In DSmT, we consider all propositions/sets in a canonical form. We take the disjunctive normal form, which is a disjunction of conjunctions, and it is unique in Boolean algebra and simplest. For example, $X=A \cap B \cap(A \cup B \cup C)$ it is not in a canonical form, but we simplify the formula and $X=A \cap B$ is in a canonical form. Like most of fusion rules ${ }^{5}$, PCR5 is not associative and the optimal fusion result is obtained by combining the sources altogether at the same time when possible. Some of PCR5 properties can be found in [1] and it allows non-Bayesian reasoning. An extension of PCR5 for combining qualitative bba's can

\footnotetext{
${ }^{3}$ i.e. the non normalized Dempster's rule.

${ }^{4}$ i.e. each source provides its bba independently of the other sources.

${ }^{5}$ Except Dempster's rule, and conjunctive rule in free DSm model.
} 
be found in [9], Vol. $2 \& 3$.

Basically, the idea of PCR5 is to transfer the conflicting mass only to the elements involved in the conflict and proportionally to their individual masses, so that the specificity of the information is entirely preserved through this fusion process. For example: consider two bba's $m_{1}($.$) and m_{2}(),. A \cap B=\emptyset$ for the model of $\Theta$, and $m_{1}(A)=0.6$ and $m_{2}(B)=0.3$. With PCR5 the partial conflicting mass $m_{1}(A) m_{2}(B)=0.6 \cdot 0.3=0.18$ is redistributed to $A$ and $B$ only with respect to the following proportions respectively: $x_{A}=0.12$ and $x_{B}=0.06$ because the proportionalization requires

$$
\frac{x_{A}}{m_{1}(A)}=\frac{x_{B}}{m_{2}(B)}=\frac{m_{1}(A) m_{2}(B)}{m_{1}(A)+m_{2}(B)}=\frac{0.18}{0.9}=0.2
$$

Variant of PCR5 (PCR6): The extension and a variant of (3), called PCR6 has been proposed by Martin and Osswald in [9], Vol. 2, for combining $s>2$ sources and for working in other fusion spaces is presented in [9]. For two sources, PCR6 coincides with PCR5. The difference between PCR5 and PCR6 lies in the way the proportional conflict redistribution is done as soon as three or more sources are involved in the fusion. For example, let's consider three sources with bba's $m_{1}(),. m_{2}($.$) and m_{3}(),. A \cap B=\emptyset$ for the model of the frame $\Theta$, and $m_{1}(A)=0.6, m_{2}(B)=0.3$, $m_{3}(B)=0.1$. With PCR5 the partial conflicting mass $m_{1}(A) m_{2}(B) m_{3}(B)=0.6 \cdot 0.3 \cdot 0.1=0.018$ is redistributed back to $A$ and $B$ only with respect to the following proportions respectively: $x_{A}^{P C R 5}=0.01714$ and $x_{B}^{P C R 5}=0.00086$ because the proportionalization requires

$$
\frac{x_{A}^{P C R 5}}{m_{1}(A)}=\frac{x_{B}^{P C R 5}}{m_{2}(B) m_{3}(B)}=\frac{m_{1}(A) m_{2}(B) m_{3}(B)}{m_{1}(A)+m_{2}(B) m_{3}(B)}
$$

that is

$$
\frac{x_{A}^{P C R 5}}{0.6}=\frac{x_{B}^{P C R 5}}{0.03}=\frac{0.018}{0.6+0.03} \approx 0.02857
$$

thus

$$
\left\{\begin{array}{l}
x_{A}^{P C R 5}=0.60 \cdot 0.02857 \approx 0.01714 \\
x_{B}^{P C R 5}=0.03 \cdot 0.02857 \approx 0.00086
\end{array}\right.
$$

With the PCR6 fusion rule, the partial conflicting mass $m_{1}(A) m_{2}(B) m_{3}(B)=0.6 \cdot 0.3 \cdot 0.1=0.018$ is redistributed back to $A$ and $B$ only with respect to the following proportions respectively: $x_{A}^{P C R 6}=0.0108$ and $x_{B}^{P C R 6}=0.0072$ because the PCR6 proportionalization is done as follows:

$\frac{x_{A}^{P C R 6}}{m_{1}(A)}=\frac{x_{B, 2}^{P C R 6}}{m_{2}(B)}=\frac{x_{B, 3}^{P C R 6}}{m_{3}(B)}=\frac{m_{1}(A) m_{2}(B) m_{3}(B)}{m_{1}(A)+m_{2}(B)+m_{3}(B)}$

that is

$$
\frac{x_{A}^{P C R 6}}{0.6}=\frac{x_{B, 2}^{P C R 6}}{0.3}=\frac{x_{B, 3}^{P C R 6}}{0.1}=\frac{0.018}{0.6+0.3+0.1}=0.018
$$

thus

$$
\left\{\begin{array}{l}
x_{A}^{P C R 6}=0.6 \cdot 0.018=0.0108 \\
x_{B C 2}^{P C R}=0.3 \cdot 0.018=0.0054 \\
x_{B, 3}^{P C R 6}=0.1 \cdot 0.018=0.0018
\end{array}\right.
$$

and therefore with PCR6, one gets finally the following redistributions to $A$ and $B$ :

$$
\left\{\begin{array}{l}
x_{A}^{P C R 6}=0.0108 \\
x_{B}^{P C R 6}=x_{B, 2}^{P C R 6}+x_{B, 3}^{P C R 6}=0.0054+0.0018=0.0072
\end{array}\right.
$$

From the implementation point of view, PCR6 is much more simple to implement than PCR5 (see Appendix).

\section{Reliability discounting}

Reliability refers to information quality while importance refers to subjective preferences of the fusion system designer. The reliability of a source represents its ability to provide the correct assessment/solution of the given problem. It is characterized by a discounting reliability factor, usually denoted $\alpha$ in $[0,1]$, which should be estimated from statistics when available, or by other techniques [3]. This reliability factor can be context-dependent. For example, if one knows that some sensors do not perform well under bad weather conditions, etc, one will decrease the reliability factor of information arising from that source accordingly. By convention, we usually take $\alpha=1$ when the source is fully reliable and $\alpha=0$ if the source is totally unreliable. Reliability of a source is generally considered ${ }^{6}$ through Shafer's discounting method [8], p. 252, which consists in multiplying the masses of focal elements by the reliability factor $\alpha$, and transferring all the remaining discounted mass to the full ignorance $\Theta$. When $\alpha<1$, such very simple reliability discounting technique discounts all focal elements with the same factor $\alpha$ and it increases the non specificity of the discounted sources since the mass committed to the full ignorance always increases. Mathematically, Shafer's discounting technique for taking into account the reliability factor $\alpha \in[0,1]$ of a given source with a bba $m($.$) and a frame$ $\Theta$ is defined by:

$$
\left\{\begin{array}{l}
m_{\alpha}(X)=\alpha \cdot m(X), \quad \text { for } X \neq \Theta \\
m_{\alpha}(\Theta)=\alpha \cdot m(\Theta)+(1-\alpha)
\end{array}\right.
$$

\section{Importance discounting}

The importance of a source is not the same as its reliability and it can be characterized by an importance factor, denoted $\beta$ in $[0,1]$. $\beta$ factor represents somehow the weight of importance granted to the source by the fusion system designer. The choice of $\beta$ is usually not related with the reliability of the source and can be chosen to any value in $[0,1]$ by the designer for his/her

\footnotetext{
${ }^{6}$ More sophisticated methods have been also proposed, see $[4,5]$ for example.
} 
own reason. By convention, the fusion system designer will take $\beta=1$ when he/she wants to grant the maximal importance of the source in the fusion process, and will take $\beta=0$ if no importance at all is granted to this source in the fusion process. Typically, if one has a pool of experts around a table to take important decision, say politicians, scientific researchers, military officers, etc, it is possible that one wants to grant more importance to the voice of a given politician (say the President) rather than to a military officers or a scientific researcher, even if the scientific researcher is more reliable in his expertise field than other people. Such situations occur frequently in real-life problems. The fusion designer must be able to deal with importance factors in a different way than with reliability factors since they correspond to distinct properties associated with a source of information.

The main question we are concerned in this paper is how to deal with different importances of sources in the fusion process in such a way that a clear distinction is made/preserved between reliability and importance?

Our preliminary investigations were based on the self/auto-combination of the sources. For example, if one has the importances factors $\beta_{1}=0.7$ for the source $s_{1}$ and $\beta_{2}=0.3$ for the source $s_{2}$, one could imagine to combine 7 times the bba $m_{1}($.$) with it-$ self, combine 3 times the bba $m_{2}($.$) with itself, and$ then combine the resulting auto-fusioned bba's because such combination would reflect somehow the relative importance of the source in the fusion process since $\beta_{1} / \beta_{2}=0.7 / 0.3=7 / 3$. Actually such approach is very disputable and cannot be used satisfactorily in practice whatever the fusion rule is adopted. It can be easily shown that the auto-conflict tends quickly to 1 after several auto-fusions [3]. In other words, the combination result of $N \times \beta_{1}$ bba's $m_{1}$ (.) with $M \times \beta_{2}$ bba's $m_{2}$ (.) is almost the same for any $N$ and $M$ sufficiently large, so that the different importances of sources are not well preserved in such approach. The numerical complexity of such method must be pointed out since it would require to compute possibly many auto-fusions of each source which is a very time-consuming computational task. For example, if $\beta_{1}=0.791$ and $\beta_{2}=0.209$, it would require to combine at least 791 auto-fusions of $m_{1}($.$) with 209$ auto-fusions of $m_{2}($.$) !!!$

In this paper, we propose a better solution to consider importances of sources. Our new approach can be considered as the dual of Shafer's discounting approach for reliabilities of sources. The idea was originally introduced briefly by Tacnet in [9], Vol.3, Chap. 23 , p. 613 . It consists to define the importance discounting with respect to the empty set rather than the total ignorance $\Theta$ (as done by Shafer in reliability discounting presented in section 3 ). Such new discounting technique allows to deal easily with sources of different importances and is also very simple to use as it will be shown.
Definition (importance discounting): We define the importance discounting of a source having the importance factor $\beta$ and asociated bba $m($.$) by$

$$
\left\{\begin{array}{l}
m_{\beta}(X)=\beta \cdot m(X), \quad \text { for } X \neq \emptyset \\
m_{\beta}(\emptyset)=\beta \cdot m(\emptyset)+(1-\beta)
\end{array}\right.
$$

Note that with this importance discounting approach, we allow to deal with non-normal bba since $m_{\beta}(\emptyset) \geq 0$. The interest of this new discounting is to preserve the specificity of the primary information since all focal elements are discounted with same importance factor and no mass is committed back to partial or total ignorances. Working with positive mass of belief on the empty set is not new and has been introduced in nineties by Smets in his transferable belief model [10]. Here we use the positive mass of the empty set as an intermediate/preliminary step of the fusion process. Clearly when $\beta=1$ is chosen by the fusion designer, it will mean that the source must take its full importance in the fusion process and so the original bba $m($.$) is$ kept unchanged. If the fusion designer takes $\beta=0$, one will deal with $m_{\beta}(\emptyset)=1$ which must be interpreted as a fully non important source. $m(\emptyset)>0$ is not interpreted as the mass committed to some conflicting information (classical interpretation), nor as the mass committed to unknown elements when working with the open-world assumption (Smets interpretation), but only as the mass of the discounted importance of a source in this particular context.

Before going further, it is worth to note that Dempster's rule cannot deal properly with importance discounted bba's proposed in (5) because our importance discounting technique preserves the specificity of the primary information and Dempster's rule does not make a difference in results when combining $m_{1}($. with $m_{2}($.$) or when combining m_{\beta_{1} \neq 1}($.$) with m_{\beta_{2} \neq 1}($. due to the way of processing the total conflicting mass of belief. This can be stated as the following theorem:

Theorem 1: Dempster's rule is not responding to the discounting of sources towards the emptyset. Proof: Let $m_{1}($.$) and m_{2}($.$) be two bba's defined$ on the fusion space $G^{\Theta}=\left\{X_{1}, X_{2}, \ldots, X_{n}\right\}$. Let $m_{1}\left(X_{i}\right)=a_{i}$ for all $i$, with $\sum_{i=1}^{n} a_{i}=1$, and all $a_{i}$ in $[0,1]$, and let $m_{2}\left(X_{i}\right)=b_{i}$ for all $i$, with $\sum_{i=1}^{n} b_{i}=1$, and all $b_{i}$ in $[0,1] . m_{1}(\emptyset)=m_{2}(\emptyset)=0$. After discounting both $m_{1}($.$) and m_{2}($.$) towards the$ emptyset with $\beta_{1}$ and respectively $\beta_{2}$ in $[0,1]$, we get: $\left(\beta_{1}\right) m_{1}\left(X_{i}\right)=\left(\beta_{1}\right) a_{i}$ for all $i$, with $\sum_{i=1}^{n} a_{i}=1$, and all $a_{i}$ in $[0,1]$, also $\left(\beta_{1}\right) m_{1}(\emptyset)=1-\beta_{1}$, and $\left(\beta_{2}\right) m_{2}\left(X_{i}\right)=\left(\beta_{2}\right) b_{i}$ for all $i$, with $\sum_{i=1}^{n} b_{i}=1$, and all $b_{i}$ in $[0,1]$, also $\left(\beta_{2}\right) m_{1}(\emptyset)=1-\beta_{2}$. If we apply the conjunctive rule to $m_{1}($.$) and m_{2}($.$) we get:$ $m_{12}\left(X_{i}\right)=c_{i}$, with $\sum_{i=1}^{n} c_{i}=1$ and $c_{i}$ in $[0,1]$, where some $X_{i}$ could be empty intersections. Suppose the 
non-empty resulted sets after applying the conjunctive rule are: $X_{i_{1}}, \ldots, X_{i_{p}}$. Then Dempster's rule gives $m_{D S}\left(X_{i_{k}}\right)=m_{12}\left(X_{i_{k}}\right) /\left(m_{12}\left(X_{i_{1}}\right)+\ldots+m_{12}\left(X_{i_{p}}\right)\right)$, for $1 \leq k \leq p$. If we apply the conjunctive rule to $\left(\beta_{1}\right) m_{1}($. and $\left(\beta_{2}\right) m_{2}($.$) we get: \left(\beta_{1}\right)\left(\beta_{2}\right) m_{12}\left(X_{i}\right)=\left(\beta_{1}\right)\left(\beta_{2}\right) c_{i}$, with $\left(\beta_{1}\right)\left(\beta_{2}\right) c_{i}$ in $[0,1]$, where some $X_{i}$ could be empty sets, and $\left(\beta_{1}\right)\left(\beta_{2}\right) m_{12}\left(\emptyset^{7}\right)=1-\left(\beta_{1}\right)\left(\beta_{2}\right)$. Now Dempster's rule normalizes the conjunctive result of non empty sets by dividing the mass of each nonempty set by the sum of all non-empty sets. The non-empty resulted sets after applying the conjunctive rule are the same: $X_{i_{1}}, \ldots, X_{i_{p}}$. Then: $\left(\beta_{1}\right)\left(\beta_{2}\right) m_{D S}\left(X_{i_{k}}\right)=$ $\left(\beta_{1}\right)\left(\beta_{2}\right) m_{12}\left(X_{i_{k}}\right) /\left(\left(\beta_{1}\right)\left(\beta_{2}\right) m_{12}\left(X_{i_{1}}\right)+\ldots+\right.$ $\left.\left(\beta_{1}\right)\left(\beta_{2}\right) m_{12}\left(X_{i_{p}}\right)\right)=m_{12}\left(X_{i_{k}}\right) /\left(m_{12}\left(X_{i_{1}}\right)+\ldots+\right.$ $\left.m_{12}\left(X_{i_{p}}\right)\right)=m_{D S}\left(X_{i_{k}}\right)$ since the whole fraction is simplified by $\left(\beta_{1}\right)\left(\beta_{2}\right)$, for $1 \leq k \leq p$. Hence Dempster's rule is not responding to the discounting of sources towards the empty set.

From Theorem 1, one understands why such importance discounting technique has never been proposed and used in DST framework and this explains why the classical Shafer's discounting technique (the reliability discounting) has only been largely adopted so far. By using Dempster's rule, the fusion designer has no other choice but to consider importance and reliability as same notions! As it will be shown, the DSmT framework with PCR5 (or PCR6) rule and the importance discounting technique proposed here provides an interesting and simple solution for the fusion of sources with different importances which makes a clear distinction between importances and reliabilities of sources.

Fusion of importance discounted bba's: Based on this new discounting technique, it is however very simple to adapt PCR5 or PCR6 fusion rules for combining the $s \geq 2$ discounted bba's associated with each source $i, i=1,2, \ldots s$. It suffices actually to consider the following extension of PCR5, denoted PCR $5_{\emptyset}$ and defined by:

- For two sources $(s=2): \forall A \in G^{\Theta}$ (A may be the empty set too)

$$
\begin{aligned}
& m_{P C R 5_{\emptyset}}(A)=\sum_{\substack{X_{1}, X_{2} \in G^{\Theta} \\
X_{1} \cap X_{2}=A}} m_{1}\left(X_{1}\right) m_{2}\left(X_{2}\right)+ \\
& \sum_{\substack{X \in G^{\Theta} \\
X \cap A=\emptyset}}\left[\frac{m_{1}(A)^{2} m_{2}(X)}{m_{1}(A)+m_{2}(X)}+\frac{m_{2}(A)^{2} m_{1}(X)}{m_{2}(A)+m_{1}(X)}\right]
\end{aligned}
$$

\footnotetext{
${ }^{7}$ i.e. the absolute empty set, not that resulted from set intersections which are empty.
}

- For $s \geq 2$ sources: $\forall A \in G^{\Theta}$ ( $A$ may be the empty set too)

$$
\begin{aligned}
& m_{P C R 5_{\emptyset}}(A)=m_{12 \ldots s}(A)+ \\
& \sum_{2 \leq t \leq s} \sum_{X_{j_{2}}, \ldots, X_{j_{t}} \in G^{\Theta}} \\
& \begin{array}{cc}
1 \leq r_{1}, \ldots, r_{t} \leq s \\
X_{j_{2}}, \ldots, X_{j_{t}} \in G^{\Theta} \\
\left\{j_{2}, \ldots, j_{t}\right\} \in \mathcal{P}^{t-1}(\{1, \ldots, n\}) \\
A \cap r_{2}<\ldots<r_{t-1}<\left(r_{t}=s\right)
\end{array} \\
& \begin{array}{c}
A \cap X_{j_{2}} \cap \ldots \cap X_{j_{s}}=\emptyset \\
\left\{i_{1}, \ldots, i_{s}\right\} \in \mathcal{P}^{s}(\{1, \ldots, s\})
\end{array} \\
& \frac{\left(\prod_{k_{1}=1}^{r_{1}} m_{i_{k_{1}}}(A)^{2}\right) \cdot\left[\prod_{l=2}^{t}\left(\prod_{k_{l}=r_{l-1}+1}^{r_{l}} m_{i_{k_{l}}}\left(X_{j_{l}}\right)\right]\right.}{\left(\prod_{k_{1}=1}^{r_{1}} m_{i_{k_{1}}}(A)\right)+\left[\sum_{l=2}^{t}\left(\prod_{k_{l}=r_{l-1}+1}^{r_{l}} m_{i_{k_{l}}}\left(X_{j_{l}}\right)\right]\right.}
\end{aligned}
$$

where $i, j, k, r, s$ and $t$ in (7) are integers. $m_{12 \ldots s}(A) \triangleq \sum_{\substack{X_{1}, X_{2}, \ldots, X_{s} \in G^{\Theta} \\ \cap_{i=1}^{s} X_{i}=A}} \prod_{i=1}^{s} m_{i}\left(X_{i}\right)$ is the conjunctive consensus on $A$ between $s$ sources and where all denominators are different from zero. If a denominator is zero, that fraction is discarded; $\mathcal{P}^{k}(\{1,2, \ldots, n\})$ is the set of all subsets of $k$ elements from $\{1,2, \ldots, n\}$ (permutations of $n$ elements taken by $k$ ), the order of elements doesn't count.

A similar extension can be done for the PCR6 formula for $s>2$ sources given in [9], Vol. 2. More precisely for any $A$ in $G^{\Theta}$ ( $A$ may be the empty set too) we define:

$$
\begin{aligned}
& m_{P C R 6_{\emptyset}}(A)=m_{12 \ldots s}(A)+ \\
& \sum_{X_{1}, X_{2}, \ldots, X_{s-1} \in G^{\Theta}} \sum_{k=1}^{s-1} \sum_{\left(i_{1}, i_{2}, \ldots, i_{s}\right) \in P(1,2, \ldots, s)} \\
& \begin{array}{c}
X_{1}, X_{2}, \ldots, X_{s-1} \in G \\
X_{i} \neq A, i \in\{1,2, \ldots, s-1\}
\end{array} \\
& \left(\cap_{i=1}^{s-1} X_{i}\right) \cap A=\emptyset \\
& {\left[m_{i_{1}}(A)+m_{i_{2}}(A)+\ldots+m_{i_{k}}(A)\right] \times} \\
& \frac{\prod_{j=1}^{k} m_{i_{j}}(A) \prod_{p=k+1}^{s-1} m_{i_{p}}\left(X_{p}\right)}{\sum_{j=1}^{k} m_{i_{j}}(A)+\sum_{p=k+1}^{s-1} m_{i_{p}}\left(X_{p}\right)}
\end{aligned}
$$

where $P(1,2, \ldots, s)$ is the set of all permutations of the elements $\{1,2, \ldots, s\}$. It should be observed that $X_{1}, X_{2}, \ldots, X_{s-1}$ may be different from each other, or some of them equal and others different, etc.

As a particular case for $s=3$ sources, one gets for any $A$ in $G^{\Theta}$ ( $A$ may be the empty set too):

$$
\begin{aligned}
& m_{P C R 6_{\emptyset}}(A)=m_{123}(A)+\sum_{\substack{X, Y \in G \Theta \\
X \neq A, Y \neq A \\
X \cap Y \cap A=\emptyset}} \sum_{\left(i_{1}, i_{2}, i_{3}\right) \in P(1,2,3)} \\
& \frac{m_{i_{1}}(A)^{2} m_{i_{2}}(X) m_{i_{3}}(Y)}{m_{i_{1}}(A)+m_{i_{2}}(X)+m_{i_{3}}(Y)} \\
& +\left[m_{i_{1}}(A)+m_{i_{2}}(A)\right] \cdot \frac{m_{i_{1}}(A) m_{i_{2}}(A) m_{i_{3}}(X)}{m_{i_{1}}(A)+m_{i_{2}}(A)+m_{i_{3}}(X)}
\end{aligned}
$$


where $m_{123}(A)$ is the mass of the conjunctive consensus on $A$ and $P(1,2,3)$ is the set of all permutations of the elements $\{1,2,3\}$. It should be observed that $X$ may be different or equal to $Y$.

The difference between formulas (3) and (6) is that $m_{P C R 5}(\emptyset)=0$ whereas $m_{P C R 5_{\emptyset}}(\emptyset) \geq 0$. Of course, since we usually need to work with normal bba's for decision-making support, the result $m_{P C R 5_{\emptyset}}($.$) , or$ $m_{P C R 6_{\emptyset}}($.$) , of the fusion of discounted masses m_{\beta_{i}}($. will be normalized by redistributing the mass of belief committed to the empty set to the other focal elements and proportionally to their masses (see next example).

\section{Example}

For convenience and simplicity, and due to space limitation constraint, we give a very simple example working on the classical power set $2^{\Theta}$ since most of readers familiar belief functions usually work with this fusion space. Example 1: Let's consider $\Theta=\{A, B\}$, Shafer's model, and two sources with respectively bba's $m_{1}($.$) and m_{2}($.$) given by m_{1}(A)=0.8, m_{1}(B)=0.2$ and $m_{2}(A)=0.4, m_{2}(B)=0.6$.

- Case 1 (no importance discounting): Let's consider that $\beta_{1}=1$ and $\beta_{2}=1$, i.e. the sources must have the same maximal importance in the fusion rule. In that case, one gets: $m_{\beta_{1}}()=$. $m_{1}($.$) and m_{\beta_{2}}()=.m_{2}($.$) and the bba's are ac-$ tually not discounted. The conjunctive rule gives $m_{12}(A)=0.32, m_{12}(B)=0.12$ and the mass $m_{12}(A \cap B=\emptyset)=0.56$ is redistributed back to $A$ and $B$ proportionally to their masses following the PCR5 principle explained in section 2. We get the following result:

\begin{tabular}{|l|cc|c|c|}
\hline & $m_{\beta_{1}=1}()$. & $m_{\beta_{2}=1}()$. & $m_{12}()$. & $m_{P C R 5}()$. \\
\hline$\emptyset$ & 0 & 0 & 0.56 & 0 \\
$A$ & 0.8 & 0.4 & 0.32 & 0.64 \\
$B$ & 0.2 & 0.6 & 0.12 & 0.36 \\
\hline
\end{tabular}

Table 1: PCR5 fusion of $m_{\beta_{1}=1}($.$) with m_{\beta_{2}=1}($.$) .$

- Case 2 (with importance discounting): Let's take now the importances factors $\beta_{1}=0.2$ and $\beta_{2}=0.8$ (note that in general we don't need to force the sum of $\beta_{i}$ to be one, unless one wants to deal with relative importances between sources). Applying importance discounting technique and normalization of $m_{P C R 5_{\emptyset}}$, denoted $m_{P C R 5_{\emptyset}}^{n o r m}($.$) , one gets:$

\begin{tabular}{|l|cc|c|c|}
\hline & $m_{\beta_{1}=0.2}()$. & $m_{\beta_{2}=0.8}()$. & $m_{12}()$. & $m_{P C R 5_{\emptyset}}^{\text {norm }}()$. \\
\hline$\emptyset$ & 0.80 & 0.20 & 0.9296 & 0 \\
$A$ & 0.16 & 0.32 & 0.0512 & 0.43 \\
$B$ & 0.04 & 0.48 & 0.0192 & 0.57 \\
\hline
\end{tabular}

Table 2: PCR5 fusion of $m_{\beta_{1}=0.2}($.$) with m_{\beta_{2}=0.8}($.$) .$
Clearly, one sees in Table 2 the strong impact of the importance discounting on the result with respect to what we obtain in Table 1 (i.e. without importance discounting). Note also that the difference is very different to what we would have obtained by taking $\alpha_{1}=0.2$ and $\alpha_{2}=0.8$ and using the reliability discounting approach as seen in Table 3 .

\begin{tabular}{|l|cc|c|c|}
\hline & $m_{\alpha_{1}=0.2}()$. & $m_{\alpha_{2}=0.8}()$. & $m_{12}()$. & $m_{P C R 5}()$. \\
\hline$\emptyset$ & 0 & 0 & 0.0896 & 0 \\
$A$ & 0.16 & 0.32 & 0.3392 & 0.3698 \\
$B$ & 0.04 & 0.48 & 0.4112 & 0.4702 \\
$A \cup B$ & 0.80 & 0.20 & 0.1600 & 0.1600 \\
\hline
\end{tabular}

Table 3: PCR5 fusion of $m_{\alpha_{1}=0.2}($.$) with m_{\alpha_{2}=0.8}($.$) .$

By comparing Table 2 with Table 3 , one sees the clear difference in results obtained by these two discounting techniques which is normal.

\section{Reliability and importance}

In this section, we examine the possibility to take into account both the reliabilities $\alpha_{i}$ and the importances $\beta_{i}$ of given sources of evidence characterized by their bba's $m_{i}(),. i=1,2, \ldots, s$. The main question is how to deal with these two distinct discounting factors since in general, but when $\alpha_{i}=\beta_{i}=1$, the reliability and importance discounting approaches do not commute. Indeed, it can be easily verified (see in next example) that $m_{\alpha_{i}, \beta_{i}}(.) \neq m_{\beta_{i}, \alpha_{i}}($.$) whenever \alpha_{i} \neq 1$ and $\beta_{i} \neq 1$. $m_{\alpha_{i}, \beta_{i}}($.$) denotes the reliability discounting of m_{i}($.$) by$ $\alpha_{i}$ followed by the importance discounting of $m_{\alpha_{i}}($. by $\beta_{i}$ which explains the notation $\alpha_{i}, \beta_{i}$ used in index. Similarly, $m_{\beta_{i}, \alpha_{i}}($.$) denotes the importance discounting$ of $m_{i}($.$) by \beta_{i}$ followed by the reliability discounting of $m_{\beta_{i}}($.$) by \alpha_{i}$. To deal both with reliabilities and importances factors and because of the non commutativity of these discountings, we propose to proceed the fusion of the sources in a three-steps process as follows:

Method 1: Step 1: Apply reliability and then importance discountings to get $m_{\alpha_{i}, \beta_{i}}(),. i=1, \ldots, s$ and combine them with $P C R 5_{\emptyset}$ or $P C R 6_{\emptyset}$ and normalize the resulting bba; Step 2: Apply importance and then reliability discountings to get $m_{\beta_{i}, \alpha_{i}}(),. i=1, \ldots, s$ and combine them with $P C R 5_{\emptyset}$ or $P C R 6_{\emptyset}$ and normalize the resulting bba; Step 3 (mixing/averaging): Combine the resulting bba's of Steps 1 and 2 using the arithmetic mean operator ${ }^{8}$.

Method 2: Another simplest method which could be useful for intermediate traceability in some applications would consist to change Steps $1 \& 2$ by Step 1': Apply reliability discounting only to get $m_{\alpha_{i}}($.$) and combine$ them with PCR5 or PCR6; Step 2': Apply importance discounting only to get $m_{\beta_{i}}($.$) and combine them with$

\footnotetext{
${ }^{8}$ Other combination rules could be used also like PCR5 or PCR6, etc., but we don't see solid justification to use them again and they require more computations than the simple arithmetic mean.
} 
$P C R 5_{\emptyset}$ or $P C R 6_{\emptyset}$ and normalize the result; Step 3' same as Step 3. Due to space limitation, only Method 1 is briefly illustrated in the following simple example.

Example 2: Let's take $\Theta=\{A, B, C\}$, Shafer's model, three sources $m_{1}(),. m_{2}($.$) and m_{3}($.$) given in next table$ and assume that their reliability factors are $\alpha_{1}=0.8$, $\alpha_{2}=0.5$, and $\alpha_{3}=0.2$ and their importance factors are $\beta_{1}=0.9, \beta_{2}=0.3$ and $\beta_{3}=0.6$.

\begin{tabular}{|l|l|l|l|}
\hline & $m_{1}()$. & $m_{2}()$. & $m_{3}()$. \\
\hline$\emptyset$ & 0 & 0 & 0 \\
$A$ & 0.8 & 0.4 & 0.1 \\
$B$ & 0 & 0.3 & 0.3 \\
$A \cup B$ & 0.1 & 0.2 & 0 \\
$C$ & 0 & 0 & 0.5 \\
$A \cup C$ & 0.1 & 0 & 0 \\
$B \cup C$ & 0 & 0.1 & 0 \\
$A \cup B \cup C$ & 0 & 0 & 0.1 \\
\hline
\end{tabular}

Table 4: Sources of evidences.

By applying reliability followed by importance discounting, and by applying importance followed by reliability discounting, one gets:

\begin{tabular}{|l|l|l|l|}
\hline & $m_{\alpha_{1}, \beta_{1}}()$. & $m_{\alpha_{2}, \beta_{2}}()$. & $m_{\alpha_{3}, \beta_{3}}()$. \\
\hline$\emptyset$ & 0.1000 & 0.7000 & 0.4000 \\
$A$ & 00.5760 & 0.0600 & 0.0120 \\
$B$ & 0 & 0.0450 & 0.0360 \\
$A \cup B$ & 0.0720 & 0.0300 & 0 \\
$C$ & 0 & 0 & 0.0600 \\
$A \cup C$ & 0.0720 & 0 & 0 \\
$B \cup C$ & 0 & 0.0150 & 0 \\
$A \cup B \cup C$ & 0.1800 & 0.1500 & 0.4920 \\
\hline
\end{tabular}

Table 5: Reliability-Importance discounting.

\begin{tabular}{|l|l|l|l|}
\hline & $m_{\beta_{1}, \alpha_{1}}()$. & $m_{\beta_{2}, \alpha_{2}}()$. & $m_{\beta_{3}, \alpha_{3}}()$. \\
\hline$\emptyset$ & 0.0800 & 0.3500 & 0.0800 \\
$A$ & 0.5760 & 0.0600 & 0.0120 \\
$B$ & 0 & 0.0450 & 0.0360 \\
$A \cup B$ & 0.0720 & 0.0300 & 0 \\
$C$ & 0 & 0 & 0.0600 \\
$A \cup C$ & 0.0720 & 0 & 0 \\
$B \cup C$ & 0 & 0.0150 & 0 \\
$A \cup B \cup C$ & 0.2000 & 0.5000 & 0.8120 \\
\hline
\end{tabular}

Table 6: Importance-Reliability discounting.

The normalized results of the $P C R 5_{\emptyset}$ fusion of $m_{\alpha_{i}, \beta_{i}}$ (.) for $i=1,2,3$ (Step 1) and PCR5 $5_{\emptyset}$ fusion of $m_{\beta_{i}, \alpha_{i}}($.$) for i=1,2,3$ (Step 2) is given in next Table 7 with their arithmetic mean $\bar{m}_{P C R 5}($.) (Step 3).

\section{Conclusions}

The proposition of two different discounting techniques is an important contribution to consider both preferences and reliability in fusion problems for decision making purposes. In this paper, we have proposed a new solution for taking into account the different importances of sources of evidence in their combination.

\begin{tabular}{|l|l|l||l|}
\hline & Step 1 & Step 2 & Step 3 \\
\hline & $m_{P C R 5_{\emptyset, \alpha, \beta} \text { norm }}()$. & $m_{P C R 5_{\emptyset, \beta, \alpha} \text { norm }}()$. & $\bar{m}_{P C R 5}()$. \\
\hline$\emptyset$ & 0 & 0 & 0 \\
$A$ & 0.5741 & 0.4927 & 0.5334 \\
$B$ & 0.0254 & 0.0244 & 0.0249 \\
$A \cup B$ & 0.0311 & 0.0464 & 0.0388 \\
$C$ & 0.0182 & 0.0182 & 0.0182 \\
$A \cup C$ & 0.0233 & 0.0386 & 0.0310 \\
$B \cup C$ & 0.0032 & 0.0032 & 0.0032 \\
$A \cup B \cup C$ & 0.3247 & 0.3765 & 0.3506 \\
\hline
\end{tabular}

Table 7: Results of Steps 1, 2 \& 3.

We have shown the clear distinction between the classical reliability discounting technique and our new importance discounting method which can be used with extensions of PCR5 and PCR6 fusion rules developed in DSmT framework. It has been shown also that Dempster's rule cannot be applied satisfactorily with this importance discounting approach contrariwise to PCR5 and PCR6 rules. The importance and reliability can now be distinguished in the fusion of sources which introduces a link with Multi-Criteria Decision Problems in the fusion of sources of information. Applications of these techniques for risk prevention against natural catastrophes in mountains are under progress and results will be published in forthcoming publications.

\section{References}

[1] J. Dezert, F. Smarandache, Non Bayesian conditioning and deconditioning, Int. Workshop on Belief Functions, Brest, France, April 2010.

[2] J. Dezert, J.-M. Tacnet, M. Batton-Hubert, F. Smarandache, Multi-criteria decision making based on DSmT-AHP, Int. Workshop on Belief Functions, Brest, France, April 2010.

[3] A. Martin, A.-L. Jousselme, C. Osswald, Conflict measure for the discounting operation on belief functions, Proc. of Fusion 2008 Int. Conference, Cologne, Germany, July 2008.

[4] D. Mercier, B. Quost, T. Denoeux, Contextual discounting of belief functions, Proc. of ECQSARU2005, pp. 552-562, Barcelona, July 2005.

[5] D. Mercier, T. Denoeux, M.-H. Masson, Refined sensor tuning in the belief function framework using contextual discounting, Proc. of IPMU2006, Vol II, pp. 1443-1450, Paris, France, July 2006.

[6] T.L. Saaty, The Analytical Hierarchy Process, McGraw Hill, New York, 1980.

[7] T.L. Saaty, Fundamentals of decision making and priority theory with the analytic hierarchy process, Vol. VI of the AHP series, RWL Publications, Pittsburgh, PA, USA. 
[8] G. Shafer, A mathematical theory of evidence, Princeton University Press, 1976.

[9] F. Smarandache, J. Dezert, Advances and applications of DSmT for information fusion (Collected works), Vols. 1-3, American Research Press, 20042009. http://www.gallup.unm.edu/ smarandache/DSmT.htm

[10] Ph. Smets Ph., The Combination of Evidence in the Transferable Belief Model, IEEE Trans. PAMI 12, pp. 447-458, 1990.

[11] J.-M. Tacnet, M. Batton-Hubert, J. Dezert, Information fusion for natural hazards in mountains, Chapter 23 of [9], Vol.3, June 2009.

[12] J.-M. Tacnet, M. Batton-Hubert, J. Dezert, A twostep fusion process for multi-criteria decision applied to natural hazards in mountains, Int. Workshop on Belief Functions, Brest, April 2010.

\section{Appendix: Matlab ${ }^{\mathrm{TM}}$ code listings for PCR5 and PCR6}

For convenience, we provide two Matlab ${ }^{\mathrm{TM}}$ routines for PCR5 and PCR6 for the fusion of $s \geq 2$ sources for working with $2^{\Theta}$, i.e. working with Shafer's model. Some adaptations need to be done to work on other fusion spaces and to work with $P C R 5_{\emptyset}$ and $P C R 6_{\emptyset}$. No verification of input is done in the routines. It is assumed that the input matrix BBA is correct, both in dimension and in content. No attempt for fast computation, nor memory optimization is done in these very simple and basic codes. The derivation of all possible combinations in the loop with combvec(Combinations, vec) instruction is a very time-consuming task when the size of the problem increases and should be done once outside the routines. The j-th column of the BBA input matrix corresponds to the (vertical) bba vector $m_{j}($.$) associated with the$ j-th source $s_{j}$. Each element of a BBA matrix is in $[0,1]$ and the sum of each column must be one. If $N$ is the cardinality of the frame $\Theta$ and if $S$ is the number of sources, then the size of the BBA input matrix is $\left.\left(\left(2^{N}\right)-1\right)\right) \times S$. Each column of the BBA matrix must use the following binary encoding of elements ${ }^{9}$ of $2^{\Theta} \backslash\{\emptyset\}$. For example, if $\Theta=\{A, B, C\}$, then binary sequence $001=A, 010=B, 011=A \cup B, \ldots$, $111=A \cup B \cup C$. These codes can be used and shared for free for research purposes only. Commercial uses of these codes, or any adaptation of them, is not allowed without written agreement of the author. The use of these codes are at the own risk of the user.

\footnotetext{
${ }^{9}$ Since one always considers normal input bba's such that $m_{j}(\emptyset)=0, j=1, \ldots S$, one doesn't need to store these values in the BBA matrix. For $P C R 5_{\emptyset}$ and $P C R 6_{\emptyset}$ however, one needs to include as first row of BBA the $m_{j}(\emptyset) \geq 0$ resulting from importance discounting of the sources and make a proper adaptation of indexes in the routines.
}

\section{File : PCR5fusion.m}

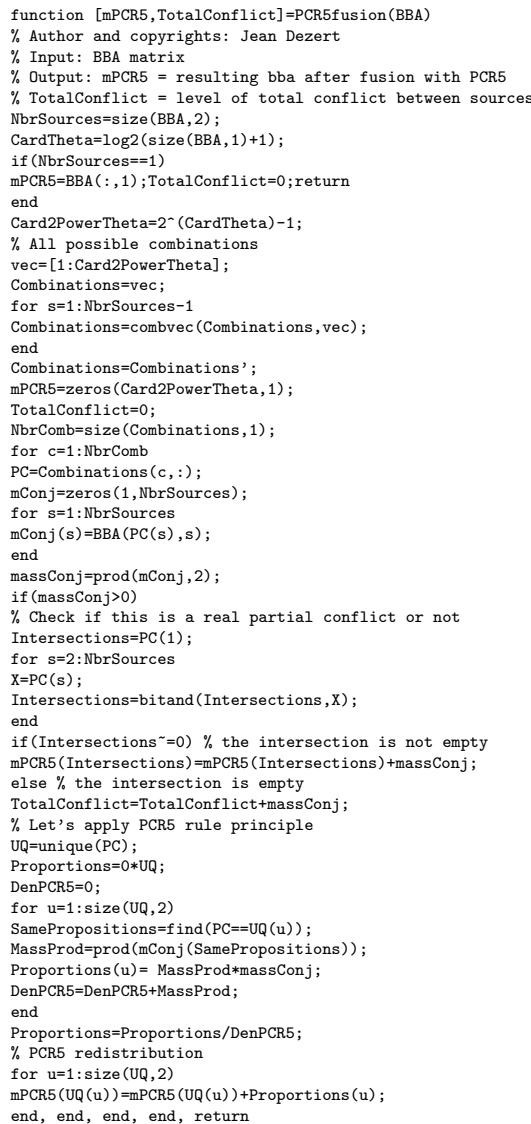

\section{File : PCR6fusion.m}

function [mPCR6, TotalConflict] =PCR6fusion (BBA) $\%$ Author and copyrights: Jean Dezert

$\%$ Input: BBA matrix

$\%$ Output: $\operatorname{mPCR} 6=$ resulting bba after fusion with PCR6 $\%$ TotalConflict $=$ level of total conflict between source NbrSources=size (BBA, 2); CardTheta $=1 \log 2(\operatorname{size}(\mathrm{BBA}, 1)+1)$ if $($ NbrSources $==1$ ) $\operatorname{mPCR} 6=\mathrm{BBA}(:, 1)$; return

end Card2PowerTheta $=2^{\wedge}$ (CardTheta) -1 ; $\%$ All possible combination vec $=[1:$ Card 2 PowerTheta $]$; Combinations $=v e c$; for $\mathrm{s}=1$ : NbrSources -1 Combinations $=$ combvec $($ Combinations,$v e c)$, end Combinations=Combinations' mPCR6=zeros (Card2PowerTheta, 1 ) NbrComb=size (Combinations, 1 ):

$\mathrm{PC}=$ Combinations (c, : ); \% particular combination mConj=zeros (1, NbrSources); for $s=1$ : NbrSources $\operatorname{mConj}(\mathrm{s})=\operatorname{BBA}(\mathrm{PC}(\mathrm{s}), \mathrm{s})$ massConj=prod (mConj , 2) ; if (massConj $>0$ ) Intersections $=\mathrm{PC}(1)$ $\mathrm{X}=\mathrm{PC}(\mathrm{s})$; Intersections=bitand (Intersections, $\mathrm{X}$ ); end if (Intersections $\left.{ }^{\sim}=0\right) \%$ intersection not empty mPCR6(Intersections) $=m$ PCR6 (Intersections) +massConj else \% empty intersection TotalConflict=lotalConflict+massConj $\%$ PCR6 rule principle Poortion $=\operatorname{mConj}(\mathrm{s}) *(\operatorname{massConj} /(\operatorname{sum}(\operatorname{mConj}, 2)))$ $\operatorname{mPCR} 6(\operatorname{PC}(\mathrm{s}))=m \operatorname{PCR} 6(\operatorname{PC}(\mathrm{s}))+$ Proportion; end, end, end, end, return 